\title{
Asymptotic solution of Sturm-Liouville problem with periodic boundary conditions for relativistic finite-difference Schrödinger equation
}

\author{
Ilkizar V. Amirkhanov ${ }^{1}$, Irina S. Kolosova ${ }^{2}$, Sergey A. Vasilyev ${ }^{2}$ \\ ${ }^{1}$ Joint Institute for Nuclear Research \\ 6, Joliot-Curie St., Dubna, Moscow Region, 141980, Russian Federation \\ 2 Peoples' Friendship University of Russia (RUDN University) \\ 6, Miklukho-Maklaya St., Moscow, 117198, Russian Federation
}

(received: July 2, 2020; accepted: September 14, 2020)

The quasi-potential approach is very famous in modern relativistic particles physics. This approach is based on the so-called covariant single-time formulation of quantum field theory in which the dynamics of fields and particles is described on a space-like three-dimensional hypersurface in the Minkowski space. Special attention in this approach is paid to methods for constructing various quasi-potentials. The quasipotentials allow to describe the characteristics of relativistic particles interactions in quark models such as amplitudes of hadron elastic scatterings, mass spectra, widths of meson decays and cross sections of deep inelastic scatterings of leptons on hadrons.

In this paper Sturm-Liouville problems with periodic boundary conditions on a segment and a positive half-line for the $2 m$-order truncated relativistic finite-difference Schrödinger equation (Logunov-Tavkhelidze-Kadyshevsky equation, LTKT-equation) with a small parameter are considered. A method for constructing of asymptotic eigenfunctions and eigenvalues in the form of asymptotic series for singularly perturbed Sturm-Liouville problems with periodic boundary conditions is proposed. It is assumed that eigenfunctions have regular and boundary-layer components. This method is a generalization of asymptotic methods that were proposed in the works of A. N. Tikhonov, A.B. Vasilyeva, and V.F Butuzov. We present proof of theorems that can be used to evaluate the asymptotic convergence for singularly perturbed problems solutions to solutions of degenerate problems when $\varepsilon \rightarrow 0$ and the asymptotic convergence of truncation equation solutions in the case $m \rightarrow \infty$. In addition, the Sturm-Liouville problem on the positive half-line with a periodic boundary conditions for the quantum harmonic oscillator is considered. Eigenfunctions and eigenvalues are constructed for this problem as asymptotic solutions for 4-order LTKT-equation.

Key words and phrases: asymptotic analysis, singularly perturbed differential equation, Sturm-Liouville problem, relativistic finite-difference Schrödinger equation, periodic boundary conditions, quasi-potential approach

(C) Amirkhanov I. V., Kolosova I. S., Vasilyev S. A., 2020

This work is licensed under a Creative Commons Attribution 4.0 International License http://creativecommons.org/licenses/by/4.0/ 


\section{Introduction}

The relativistic finite-difference analog of the Schrödinger equation (Logunov-Tavkhelidze-Kadyshevsky equation, LTK-equation) with the quasipotential in the relativistic configurational space for the radial wave functions of bound states for two identical elementary particles without spin has the form [1]-[13]:

$$
\begin{gathered}
{\left[H_{0}^{\mathrm{rad}}+V(r)-2 c \sqrt{q^{2}+m^{2} c^{2}}\right] \psi(r, l)=0} \\
H_{0}^{\mathrm{rad}}=2 m c^{2} \operatorname{ch}\left(\frac{i \hbar}{m c} D\right)+\frac{\hbar^{2} l(l+1)}{m r\left(r+\frac{i \hbar}{m c}\right)} \exp \left(\frac{i \hbar}{m c} D\right),
\end{gathered}
$$

where $m$ is a mass, $q$ is a momentum, $l$ is an angular momentum of each elementary particle and $V(r)$ is a quasi-potential (a piecewise continuous function).

Asymptotic solutions in the form of regular and boundary layer parts of boundary value problems for LTK-equation with the quasi-potential on a segment and on a positive half-line were constructed in the works [14]-[16], and the question of the asymptotic behavior of the solutions was investigated when a small parameter $\varepsilon \rightarrow 0$. Also in these works the truncation method was applied to LTK-equation. Thus, LTK-equation of infinite order was reduced to the equation of finite $2 m$-order. Boundary value problems on a segment and on a positive half-line were formulated for this "truncated" equation (Logunov-Tavkhelidze-Kadyshevsky truncated equation, LTKTequation). Eigenfunctions and eigenvalues in the form of asymptotic series were constructed for these problems and the solution behavior was studied when the order of LTKT-equation tends to infinity $2 m \rightarrow \infty$.

In the paper [17] mass spectra and probabilities of radiative decays of heavy quarkonia were obtained in the framework of the constituent quark model of hadrons based on the relativistic Logunov-Tavkhelidze-Kadyshevsky equation.

Researchers pay a lot of attention to the description of quantum systems that consist of one-dimensional linear chains of $n$ identical harmonic oscillators with a nearest neighbor interaction. Periodic boundary conditions, where the $n$-th oscillator is coupled back to the first oscillator, and fixed wall boundary conditions, where the first oscillator and the $n$-th oscillator are coupled to a fixed wall, was considered in the paper [18], [19].

In this paper Sturm-Liouville problems with periodic boundary conditions on a segment and a positive half-line are formulated for the truncated to order $2 m$ relativistic finite-difference Schrödinger equation (LogunovTavkhelidze-Kadyshevsky equation, LTKT-equation) with a small parameter.

For these singularly perturbed problems a method is proposed for constructing eigenfunctions and eigenvalues in the form of asymptotic series. This method allows to obtain asymptotic solutions in the form of regular and boundary-layer parts. It is also possible to investigate the question of asymptotic solutions behavior when $\varepsilon \rightarrow 0$ and $2 m \rightarrow \infty$. The Sturm-Liouville problem for 4-order LTKT-equation on a positive half-line with periodic 
boundary conditions is formulated for the quantum harmonic oscillator quasipotential and eigenfunctions and eigenvalues in the form of asymptotic series are constructed.

\section{The Sturm-Liouville problems for the LTKT-equation}

We consider the quasi-potential equation [3]-[5] in a relativistic configuration space for the radial wave functions of bounded states for two identical elementary particles

$$
\begin{gathered}
{\left[H_{0}^{\mathrm{rad}}+V(r)-2 c \sqrt{q^{2}+m^{2} c^{2}}\right] \psi(r, l)=0,} \\
H_{0}^{\mathrm{rad}}=2 m c^{2} \operatorname{ch}\left(\frac{i \hbar}{m c} D\right)+\frac{\hbar^{2} l(l+1)}{m r\left(r+\frac{i \hbar}{m c}\right)} \exp \left(\frac{i \hbar}{m c} D\right)= \\
=\sum_{p=0}^{\infty} \frac{(-1)^{p} 2 m c^{2}}{(2 p) ! !}\left(\frac{\hbar}{m c}\right)^{2 p} D^{2 p}+\frac{\hbar^{2} l(l+1)}{m r\left(r+\frac{i \hbar}{m c}\right)} \sum_{p=0}^{\infty} \frac{1}{p !}\left(\frac{i \hbar}{m c}\right)^{p} D^{p}, \\
D^{p}=\frac{d^{p}}{d r^{p}},
\end{gathered}
$$

where $m$ is a mass, $q$ is a momentum, $l$ is a moment of elementary particles and $V(r)$ is a quasi-potential.

We can limit the speed of light to the infinity $(c \rightarrow \infty)$ formally. In this case, the equation (1) becomes the non-relativistic Schrödinger equation [20]

$$
\left[-\hbar^{2} D^{2}+\hbar^{2} l(l+1) / r^{2}+m V(r)-q^{2}\right] \psi(r)=0 .
$$

Let physical parameter be $\hbar=1, m=1, \varepsilon=\frac{1}{c}$ and $l=0$ (case of $S$-wave) in (1) where

$$
\lambda_{\varepsilon, \infty}=2 q^{2} / \sqrt{1+\varepsilon^{2} q^{2}}+1, v=V(r), \quad q^{2}=\left(1+0.25 \varepsilon^{2} \lambda_{\varepsilon, \infty}\right) \lambda_{\varepsilon, \infty} .
$$

We can rewrite the equation (1) in the form as under

$$
\begin{gathered}
{\left[\widetilde{L}_{\infty}^{\varepsilon}-\lambda_{\varepsilon, \infty}\right] \psi_{\varepsilon, \infty}(r)=0} \\
\widetilde{L}_{\infty}^{\varepsilon}=L_{2}+\varepsilon^{2} \\
L_{\infty}^{\varepsilon}=\sum_{p=1}^{\infty} \varepsilon^{2 p-2} L_{2 p}+v(r), \quad L_{2 p}=\frac{2(-1)^{p}}{(2 p) ! !} D^{2 p}, \quad \varepsilon \in(0,1] \\
L_{2}=L_{2}+v(r)=-D^{2}+v(r) \\
L_{\infty}^{\varepsilon}=\sum_{p=1}^{\infty} \varepsilon^{2 p-2} L_{2 p+2}=\sum_{p=1}^{\infty} \frac{2(-1)^{p+1}}{(2 p+2) ! !} \varepsilon^{2 p-2} D^{2 p+2}
\end{gathered}
$$


The equation (1) is an infinite order differential equation with a small parameter $(\varepsilon \ll 1)$ at higher derivatives and we can classify it as singularly perturbed equations.

We can truncate the equation (4) to a finite equation of $2 m$-order with $m>1$ and it can be rewritten as follows

$$
\begin{gathered}
{\left[\widetilde{L}_{2 m}^{\varepsilon}-\lambda_{\varepsilon, 2 m}\right] \psi_{\varepsilon, 2 m}(r)=0,} \\
\widetilde{L}_{2 m}^{\varepsilon}=L_{2}+\varepsilon^{2} L_{2 m}^{\varepsilon}=\sum_{p=1}^{m} \varepsilon^{2 p-2} L_{2 p}+v(r), \\
L_{2 m}^{\varepsilon}=\sum_{p=1}^{m-1} \varepsilon^{2 p-2} L_{2 p+2}=\sum_{p=1}^{m-1} \frac{2(-1)^{p+1}}{(2 p+2) ! !} \varepsilon^{2 p-2} D^{2 p+2},
\end{gathered}
$$

where $L_{2}$ is the self-adjoint 2-order elliptic operator, $\widetilde{L}_{2 m}^{\varepsilon}$ is the self-adjoint $2 m$-order elliptic operator, $\psi_{\varepsilon, 2 m}(r)$ is the solution of the $2 m$-order equation.

We can formulate the boundary value problem $A_{\varepsilon}^{2 m}$ on a segment $\left[0, r_{0}\right]$ and the boundary value problem $B_{\varepsilon}^{2 m}$ on a positive half-line $[0,+\infty)$ for defining the eigenfunctions $\left[\psi_{\varepsilon, 2 m, \gamma}\right]_{\gamma=1}^{\infty}$ and the eigenvalues $\left[\lambda_{\varepsilon, 2 m, \gamma}\right]_{\gamma=1}^{\infty}$ for this differential equation as follows

$$
\left[\widetilde{L}_{2 m}-\lambda_{\varepsilon, 2 m}\right] \psi_{\varepsilon, 2 m}(r)=0
$$

where

$$
D^{i} \psi_{\varepsilon, 2 m}(0)=D^{i} \psi_{\varepsilon, 2 m}\left(r_{0}\right), \quad i=0,1, \ldots, 2 m-1,
$$

are the periodic boundary conditions of the problem $A_{\varepsilon}^{2 m}$, and

$$
D^{i} \psi_{\varepsilon, 2 m}(0)=D^{i} \psi_{\varepsilon, 2 m}(+\infty), \quad i=0,1, \ldots, 2 m-1,
$$

are the periodic boundary conditions of the problem $B_{\varepsilon}^{2 m}$.

If we assume $\varepsilon=0$, we can get the degenerate problems $A_{0}$ and $B_{0}$ for defining the eigenfunctions $\left[\psi_{0, \gamma}\right]_{\gamma=1}^{\infty}$ and the eigenvalues $\left[\lambda_{0, \gamma}\right]_{\gamma=1}^{\infty}$ of following type as under

$$
\left[L_{2}-\lambda_{0}\right] \psi_{0}(r)=0
$$

where

$$
D^{i} \psi_{0}(0)=D^{i} \psi_{0}\left(r_{0}\right), \quad i=0,1
$$

is the periodic boundary conditions of the problem $A_{0}$, and

$$
D^{i} \psi_{0}(0)=D^{i} \psi_{0}(+\infty), \quad i=0,1,
$$

is the periodic boundary conditions of the problem $B_{0}$.

We can consider the question of the behavior of the eigenfunctions $\left[\psi_{\varepsilon, 2 m, \gamma}\right]_{\gamma=1}^{\infty}$ and the eigenvalues $\left[\lambda_{\varepsilon, 2 m, \gamma}\right]_{\gamma=1}^{\infty}$ of the problems $A_{\varepsilon}^{2 m}$ and $B_{\varepsilon}^{2 m}$ in the case when a small parameter tends to zero $(\varepsilon \rightarrow 0)$ but fixed order $2 m$ of the operator $\widetilde{L}_{2 m}$, and in the case when the order $m$ is increased but a small parameter $\varepsilon$ is fixed. 
The eigenfunctions $\left[\psi_{\varepsilon, 2 m, \gamma}\right]_{\gamma=1}^{\infty}$ and $\left[\psi_{0, \gamma}\right]_{\gamma=1}^{\infty}$ are the solutions of the corresponding problems $A_{\varepsilon}^{2 m}, A_{0}$ and $B_{\varepsilon}^{2 m}, B_{0}$. These solutions are elements of a Hilbert space $H\left(\Omega_{\Gamma}\right)$ with an inner product $(\psi, \varphi)_{H\left(\Omega_{\Gamma}\right)}=\int_{\Omega_{\Gamma}} \psi(r) \varphi(r) d r$ $\left(\psi, \varphi \in H\left(\Omega_{\Gamma}\right)\right)$, in which there is a set of a linear continuous self-adjoint operators $A\left(\Omega_{\Gamma}\right): H\left(\Omega_{\Gamma}\right) \rightarrow H\left(\Omega_{\Gamma}\right)$ of problems $A_{\varepsilon}^{2 m}, B_{\varepsilon}^{2 m}, A_{0}, B_{0}\left(\widetilde{L}_{2 m}^{\varepsilon}, L_{2} \in A\right.$, $m>2$ ), where $\Omega_{\Gamma}(\Gamma=A, B)$ is a domain of the operator (a subscript $A$ corresponds to a segment $\left[0, r_{0}\right]$ and a subscript $B$ is a positive half-line $[0,+\infty))$.

Let $\left\|A\left(\Omega_{\Gamma}\right)\right\|_{H}$ denotes the norm of operators $A\left(\Omega_{\Gamma}\right)$ and we can write

$$
\left\|A\left(\Omega_{\Gamma}\right)\right\|_{H}=\sup _{\psi \in H, \psi \neq 0} \frac{\|A \psi\|_{H}}{\|\psi\|_{H}}, \quad\|\psi\|_{H}=(\psi, \psi)_{H}^{1 / 2} .
$$

We can give the sufficient conditions for the solvability of the problems $A_{0}$, $B_{0}$ and $A_{\varepsilon}^{2 m}, B_{\varepsilon}^{2 m}$.

Condition 1. The operator $L_{2}$ for the periodic boundary conditions of the problems $A_{0}$ or $B_{0}$ must be positively defined, i.e.

$$
\left(L_{2}\left(\psi_{0}\right), \psi_{0}\right)_{H\left(\Omega_{\Gamma}\right)}=\int_{\Omega_{\Gamma}} L_{2}\left(\psi_{0}\right) \psi_{0} d r=\int_{\Omega_{\Gamma}}\left|D \psi_{0}\right|^{2} d r+\int_{\Omega_{\Gamma}} v(r) \psi_{0}^{2} d r \geqslant 0,
$$

for any functions $v(r) \in C^{\infty}(\Gamma)$ and $\psi_{0} \in H\left(\Omega_{\Gamma}\right)$ from domain $\Omega_{\Gamma}$, and it must satisfy the boundary conditions of the corresponding degenerate problems $\left(A_{0}\right.$ or $\left.B_{0}\right)$.

Condition 2. The operator $L_{2 m}^{\varepsilon}$ under boundary conditions of problems $A_{\varepsilon}^{2 m}$ or $B_{\varepsilon}^{2 m}$ must be positive, i.e.

$$
\begin{aligned}
\left(L_{2 m}^{\varepsilon} \psi_{\varepsilon, 2 m}, \psi_{\varepsilon, 2 m}\right)_{H\left(\Omega_{\Gamma}\right)} & =\sum_{p=1}^{m-1} \frac{2(-1)^{p+1}}{(2 p+2) ! !} \varepsilon^{2 p-2} \int_{\Omega_{\Gamma}}\left(D^{2 p+2} \psi_{\varepsilon, 2 m}\right) \psi_{\varepsilon, 2 m} d r= \\
& =\sum_{p=1}^{m-1} \frac{2}{(2 p+2) ! !} \varepsilon^{2 p-2} \int_{\Omega_{\Gamma}}\left|D^{p+1} \psi_{\varepsilon, 2 m}\right|^{2} d r \geqslant 0
\end{aligned}
$$

for any functions $\psi_{\varepsilon, 2 m} \in H\left(\Omega_{\Gamma}\right)$ from domain $\Omega_{\Gamma}$, and it must satisfy the boundary conditions of the corresponding singularly perturbed problem $\left(A_{\varepsilon}^{2 m}\right.$ or $\left.B_{\varepsilon}^{2 m}\right)$.

It is known that the degeneration of the problems $A_{\varepsilon}^{2 m}, B_{\varepsilon}^{2 m}$ into the problems $A_{0}, B_{0}$ are regular if the number of roots with negative real parts and positive real parts of an additional characteristic equation, which in our case has the form

$$
P\left(\alpha^{2 m}\right)=\sum_{p=1}^{m} \frac{(-1)^{p}}{(2 p) ! !}\left(\alpha^{2 m}\right)^{2 p-2}=0
$$


coincide with the number of boundary conditions that drop down on the left and, respectively, on the right when we replace the consideration problems $A_{\varepsilon}^{2 m}, B_{\varepsilon}^{2 m}$ to problems $A_{0}, B_{0}$.

Let's now consider the generalized characteristic form of the operator $\sum_{p=1}^{m} \varepsilon^{2 p-2} L_{2 p}$, which is obtained by replacing $D^{2 p}$ with $(i \xi)^{2 p}$

$$
\pi_{\varepsilon}(\xi)=\sum_{p=1}^{m} \frac{2(-1)^{p}}{(2 p) ! !} \varepsilon^{2 p-2}(i \xi)^{2 p}
$$

The regular degeneration of the problems $A_{\varepsilon}^{2 m}, B_{\varepsilon}^{2 m}$ to $A_{0}, B_{0}$ is fulfilled if the following condition is true.

Condition 3. If the following inequality take place for the real part of the sum $\pi_{\varepsilon}(\xi)$

$$
\boldsymbol{\operatorname { R e }}\left(\pi_{\varepsilon}(\xi)\right)=\sum_{p=1}^{m} \frac{2}{(2 p) ! !} \varepsilon^{2 p-2} \xi^{2 p} \geqslant C \sum_{p=1}^{m} \varepsilon^{2 p-2}|\xi|^{2 p} \geqslant 0,
$$

where $C$ is not depended on $\xi$, then problems $A_{\varepsilon}^{2 m}$ and $B_{\varepsilon}^{2 m}$ degenerate into problems $A_{0}$ and $B_{0}$ regularly.

Let's assume that a set of eigenvalues $\lambda_{\varepsilon, 2 m, 1} \leqslant \lambda_{\varepsilon, 2 m, 2} \leqslant \ldots \leqslant \lambda_{\varepsilon, 2 m, n} \leqslant \ldots$ and $\lambda_{0,1} \leqslant \lambda_{0,2} \leqslant \ldots \leqslant \lambda_{0, n} \leqslant \ldots$ is ordered in ascending order $\left[\lambda_{\varepsilon, 2 m, \gamma}\right]_{\gamma=1}^{\infty}$, $\left[\lambda_{0, \gamma}\right]_{i=1}^{\infty}$, and this set of eigenvalues corresponds to a complete orthonormal set of eigenfunctions $\left[\psi_{\varepsilon, 2 m, \gamma}\right]_{\gamma=1}^{\infty},\left[\psi_{0, \gamma}\right]_{\gamma=1}^{\infty}$.

Since existence domains $\Omega_{A}$ of operators $\widetilde{L}_{2 m}$ and $L_{2}$ coincide for the problems $A_{\varepsilon}^{2 m}$ and $A_{0}$ and also for any function $\psi_{\varepsilon, 2 m} \in \Omega_{A}$, that satisfies the boundary conditions of the problem $A_{\varepsilon}^{2 m}$, the following inequality from Condition 2

$$
\left(L_{2 m}^{\varepsilon} \psi_{\varepsilon, 2 m}, \psi_{\varepsilon, 2 m}\right)_{H\left(\Omega_{A}\right)} \geqslant\left(L_{2} \psi_{\varepsilon, 2 m}, \psi_{\varepsilon, 2 m}\right)_{H\left(\Omega_{A}\right)},
$$

holds true, then the following estimate inequality occurs $\lambda_{\varepsilon, 2 m, \gamma} \geqslant \lambda_{0, \gamma}$, $\gamma=1,2, \ldots$

A similar estimate takes place for the problems $B_{\varepsilon}^{2 m}$ and $B_{0}$.

\section{Constructing of asymptotic solutions for boundary value problems}

\subsection{General scheme for constructing of the asymptotics. Regular and boundary series}

We can use methods of the singular perturbations theory of differential equations and find solutions to problems $A_{\varepsilon}^{2 m}$ and $B_{\varepsilon}^{2 m}$.

Let's search for a formal solution $\psi_{\varepsilon, 2 m}(r)$ of the problems $A_{\varepsilon}^{2 m}$ and $B_{\varepsilon}^{2 m}$ in the form of asymptotic series 


$$
\begin{aligned}
\Theta \psi_{\varepsilon, 2 m}(r)=\bar{\psi}_{2 m}(r, \varepsilon) & +\Pi_{2 m} \psi\left(\rho_{1}, \varepsilon\right)+Q_{2 m} \psi\left(\rho_{2}, \varepsilon\right)= \\
& =\sum_{k=0}^{\infty} \varepsilon^{k}\left(\bar{\psi}_{2 m, k}(r)+\Pi_{2 m, k} \psi\left(\rho_{1}\right)+Q_{2 m, k} \psi\left(\rho_{2}\right)\right)
\end{aligned}
$$

where a partial sum

$$
\Theta_{j} \psi_{\varepsilon, 2 m}(r)=\sum_{k=0}^{j} \varepsilon^{k}\left(\bar{\psi}_{2 m, k}(r)+\Pi_{2 m, k} \psi\left(\rho_{1}\right)+Q_{2 m, k} \psi\left(\rho_{2}\right)\right)
$$

satisfies inequalities for solutions of the problem $A_{\varepsilon}^{2 m}$

$$
\max _{r \in\left[\delta_{A}, r_{0}-\delta_{A}\right]}\left|\psi_{\varepsilon, 2 m}-\Theta_{j} \psi_{\varepsilon, 2 m}\right|<M_{A} \varepsilon^{j+1},
$$

and the problem $B_{\varepsilon}^{2 m}$

$$
\max _{r \in\left[\delta_{B}, \infty+\right)}\left|\psi_{\varepsilon, 2 m}-\Theta_{j} \psi_{\varepsilon, 2 m}\right|<M_{B} \varepsilon^{j+1},
$$

and similar inequalities for the boundary conditions of these problems, where $M_{A}, M_{B}$ and $\delta_{A} \ll 1, \delta_{B} \ll 1$ are positive constants that are independent of $r$ and $\varepsilon$.

The asymptotic solution for $\psi_{\varepsilon, 2 m}$ have the form as under

$$
\begin{gathered}
\psi_{\varepsilon, 2 m}(r)=\sum_{k=0}^{j} \varepsilon^{k}\left(\bar{\psi}_{2 m, k}(r)+\Pi_{2 m, k} \psi\left(\rho_{1}\right)+Q_{2 m, k} \psi\left(\rho_{2}\right)\right)+\bar{z}_{j}^{2 m}(r), \\
\bar{z}_{j}^{2 m}(r)=\psi_{\varepsilon, 2 m}-\Theta_{j} \psi_{\varepsilon, 2 m},
\end{gathered}
$$

where $\bar{z}_{j}^{2 m}(r)=\varepsilon^{j+1} z_{j}^{2 m}(r)$ is error of the asymptotic approximation of the solution $\psi_{\varepsilon, 2 m}$ by a partial sum $\Theta_{j} \psi_{\varepsilon, 2 m}$.

We can write the regular part of the asymptotic expansion in the form

$$
\bar{\psi}_{2 m}(r, \varepsilon) \equiv \bar{\psi}_{2 m, 0}(r)+\varepsilon \bar{\psi}_{2 m, 1}(r)+\varepsilon^{2} \bar{\psi}_{2 m, 2}(r)+\ldots,
$$

and the singular parts of the asymptotic expansion have the forms as under

$$
\Pi_{2 m} \psi\left(\rho_{1}, \varepsilon\right) \equiv \Pi_{2 m, 0} \psi\left(\rho_{1}\right)+\varepsilon \Pi_{2 m, 1} \psi\left(\rho_{1}\right)+\varepsilon^{2} \Pi_{2 m, 2} \psi\left(\rho_{1}\right)+\ldots,
$$

for describing the behavior of the solution on the left edge of a segment $\left[0, r_{0}\right]$ or a positive half-line $[0,+\infty)$,

$$
Q_{2 m} \psi\left(\rho_{2}, \varepsilon\right) \equiv Q_{2 m, 0} \psi\left(\rho_{2}\right)+\varepsilon Q_{2 m, 1} \psi\left(\rho_{2}\right)+\varepsilon^{2} Q_{2 m, 2} \psi\left(\rho_{2}\right)+\ldots
$$

for describing the behavior of the solution of the problem $A_{\varepsilon}^{2 m}$ on the right edge of a segment $\left[0, r_{0}\right]$.

It is known that the function $Q_{2 m} \psi\left(\rho_{2}, \varepsilon\right)=0$ for the problem $B_{\varepsilon}^{2 m}$, since the solution of the problem $B_{0}$ is chosen so that it tends to zero when $r \rightarrow+\infty$ together with all its derivatives. Here we use new independent (stretched) 
variables $\rho_{1}=r / \varepsilon$ and $\rho_{2}=\left(r_{0}-r\right) / \varepsilon$ for the boundary functions $\Pi_{2 m, k} \psi$, $Q_{2 m, k} \psi$.

Similarly, we can present the simple eigenvalue of $\lambda_{\varepsilon, 2 m}$ in the form of the asymptotic series in powers of the small parameter $\varepsilon$ in the form as under

$$
\lambda_{\varepsilon, 2 m} \equiv \lambda_{2 m, 0}+\varepsilon \lambda_{2 m, 1}+\varepsilon^{2} \lambda_{2 m, 2}+\ldots,
$$

where the partial sum

$$
\Theta_{j} \lambda_{\varepsilon, 2 m}=\sum_{k=0}^{j} \varepsilon^{k} \lambda_{2 m, k},
$$

satisfies the condition $\left|\lambda_{\varepsilon, 2 m}-\Theta_{j} \lambda_{\varepsilon, 2 m}\right|<\tilde{M} \varepsilon^{j+1}$, where $\tilde{M}>0$ is a positive constant that is independent of $r$ and $\varepsilon$.

So an asymptotic approximation of the eigenvalue $\lambda_{\varepsilon, 2 m}$ has the form as under

$$
\lambda_{\varepsilon, 2 m}=\sum_{k=0}^{j} \varepsilon^{k} \lambda_{2 m, k}+\bar{\Delta}_{j}^{2 m},
$$

where $\bar{\Delta}_{j}^{2 m}=\varepsilon^{j+1} \Delta_{j}^{2 m}$, bar $\Delta_{j}^{2 m}=\lambda_{\varepsilon, 2 m}-\Theta_{j} \lambda_{\varepsilon, 2 m}$ is an error of the asymptotic approximation of the eigenvalue $\lambda_{\varepsilon, 2 m}$ for this partial sum.

In addition, we assume that the function $v(r)$ can be decomposed as a convergent series in the neighborhood of the points $r=0$ and $r=r_{0}$

$$
v(r)=\sum_{s=-1}^{\infty} v_{s}^{1} r^{s}, \quad v(r)=\sum_{s=-1}^{\infty} v_{s}^{2}\left(r-r_{0}\right)^{s},
$$

and

$$
v\left(\rho_{1}\right)=\sum_{s=-1}^{\infty} v_{s}^{1} \varepsilon^{s} \rho_{1}^{s}, \quad v\left(\rho_{2}\right)=\sum_{s=-1}^{\infty}(-1)^{|s|} v_{s}^{2} \varepsilon^{s} \rho_{2}^{s},
$$

where $\rho_{1}=r / \varepsilon$ and $\rho_{2}=\left(r_{0}-r\right) / \varepsilon$ are the stretched variables.

\subsection{The main terms of the asymptotic series}

We can determine the terms of the asymptotic series of the decomposition $\bar{\psi}_{2 m, k}, \Pi_{2 m, k} \psi, Q_{2 m, k} \psi$ and $\lambda_{2 m, k}$ of the problems $A_{\varepsilon}^{2 m}$ and $B_{\varepsilon}^{2 m}$ if we substitute the decomposition (11), (12) and (13) in the equation (5) and the boundary conditions (6) of the problem $A_{\varepsilon}^{2 m}$ and the equation (5) and the boundary conditions ( 7 ) of the problem $B_{\varepsilon}^{2 m}$, and then we equate all members of the series that stand at equal powers of a small parameter $\varepsilon$.

We should use additional requirements for the boundary functions

$$
\Pi_{2 m, k} \psi\left(\rho_{1}\right) \rightarrow 0, \quad Q_{2 m, k} \psi\left(\rho_{2}\right) \rightarrow 0, \quad k=0,1,2, \ldots,
$$

where $\varepsilon \rightarrow 0$ and a fixed $r$. These requirements allows to select the solutions $\Pi_{2 m, k} \psi$ and $Q_{2 m, k} \psi$ that tend to zero outside the boundary layer only. 


\subsubsection{Building a zero approximation of the asymptotic expansion}

We can get the systems of equations and determine the solutions $\bar{\psi}_{2 m, 0}$, $\Pi_{2 m, 0} \psi, Q_{2 m, 0} \psi$ and $\lambda_{2 m, 0}$ of the problems $A_{\varepsilon}^{2 m}$ and $B_{\varepsilon}^{2 m}$ in a zero approximation in the form

$$
\begin{gathered}
{\left[L_{2}-\lambda_{2 m, 0}\right] \bar{\psi}_{2 m, 0}=0, \quad L_{2}=-D^{2}+v(r),} \\
L_{2 m}^{1} \Pi_{2 m, 0} \psi=0, \quad L_{2 m}^{1}=\sum_{p=1}^{m} \frac{2(-1)^{p}}{(2 p) ! !} \frac{d^{2 p}}{d \rho_{1}^{2 p}}, \\
L_{2 m}^{2} Q_{2 m, 0} \psi=0, \quad L_{2 m}^{2}=\sum_{p=1}^{m} \frac{2(-1)^{p}}{(2 p) ! !} \frac{d^{2 p}}{d \rho_{2}^{2 p}}, \\
D^{i}\left(\bar{\psi}_{2 m, 0}(0)+\Pi_{2 m, 0} \psi(0)\right)=D^{i}\left(\bar{\psi}_{2 m, 0}(\bar{r})+Q_{2 m, 0} \psi(\bar{r})\right), \\
\Pi_{2 m, 0} \psi\left(\rho_{1}\right) \rightarrow 0, \quad Q_{2 m, 0} \psi\left(\rho_{2}\right) \rightarrow 0, \quad \varepsilon \rightarrow 0, \quad i=0,1,2, \ldots, 2 m-1,
\end{gathered}
$$

where $\bar{r}=r_{0}$ for $A_{\varepsilon}^{2 m}$ and $\bar{r} \rightarrow+\infty$ for $B_{\varepsilon}^{2 m}$.

The eigenfunctions $\left[\bar{\psi}_{2 m, 0, \gamma}\right]_{\gamma=1}^{\infty}$ and the eigenvalues $\left[\lambda_{2 m, 0, \gamma}\right]_{\gamma=1}^{\infty}$ coincide with the solutions of the corresponding degenerate problems $A_{0}$ or $B_{0}$.

Thus, we can determine the boundary functions $\Pi_{2 m, 0} \psi\left(\rho_{1}\right), Q_{2 m, 0} \psi\left(\rho_{2}\right)$ if we find the solutions of the boundary value problems as under

$$
\begin{gathered}
L_{2 m}^{1} \Pi_{2 m, 0} \psi=0, \quad L_{2 m}^{2} Q_{2 m, 0} \psi=0, \\
D^{i} \Pi_{2 m, 0} \psi(0)+D^{i} \bar{\psi}_{2 m, 0}(0)=D^{i} Q_{2 m, 0} \psi(\bar{r})+D^{i} \bar{\psi}_{2 m, 0}(\bar{r}), \\
\Pi_{2 m, 0} \psi\left(\rho_{1}\right) \rightarrow 0, \quad Q_{2 m, 0} \psi\left(\rho_{2}\right) \rightarrow 0, \quad \varepsilon \rightarrow 0, \quad i=0,1,2, \ldots, 2 m-1 .
\end{gathered}
$$

We can write the functions $\Pi_{2 m, 0} \psi\left(\rho_{1}\right)$ and $Q_{2 m, 0} \psi\left(\rho_{2}\right)$ in the forms

$$
\begin{aligned}
\Pi_{2 m, 0} \psi\left(\rho_{1}\right) & =\sum_{\zeta=1}^{m-1} C_{\zeta, 0}^{2 m, 1} \exp \left(-\alpha_{\zeta}^{2 m} \rho_{1}\right), \\
Q_{2 m, 0} \psi\left(\rho_{2}\right) & =\sum_{\zeta=1}^{m-1} C_{\zeta, 0}^{2 m, 2} \exp \left(-\alpha_{\zeta}^{2 m} \rho_{2}\right) .
\end{aligned}
$$

Hence, the number of arbitrary constants $C_{\zeta, 0}^{2 m, 1}$ and $C_{\zeta, 0}^{2 m, 2}$ equals the number of disappearing boundary conditions of problems $A_{\varepsilon}^{2 m}$ or $B_{\varepsilon}^{2 m}$ when we try formulate the degenerate problems $A_{0}$ or $B_{0}$.

Let the values $\alpha_{\zeta}^{2 m}(\zeta=1, \ldots, 2 m-2)$ be the roots of the additional characteristic equation

$$
P\left(\alpha^{2 m}\right)=\sum_{p=1}^{m} \frac{(-1)^{p}}{(2 p) ! !}\left(\alpha^{2 m}\right)^{2 p-2}=0 .
$$


Since an algebraic equation

$$
\boldsymbol{\operatorname { R e }}\left(\alpha_{\zeta}^{2 m}\right)>0, \quad \zeta=\overline{1, m-1}, \quad \boldsymbol{\operatorname { R e }}\left(\alpha_{\zeta}^{2 m}\right)<0, \quad \zeta=\overline{m, 2 m-2},
$$

is biquadrate; thus, it has the same number of roots with positive and negative real parts.

We can get the following relations from the boundary conditions

$$
D^{i} \Pi_{2 m, 0} \psi(0)-D^{i} Q_{2 m, 0} \psi(\bar{r})=-D^{i} \bar{\psi}_{2 m, 0}(0)+D^{i} \bar{\psi}_{2 m, 0}(\bar{r}),
$$

where $i=0,1,2, \ldots, 2 m-1$, and we can derive a system of $2 m$ linear equations like that

$$
\mathbf{D}^{2 m} \overrightarrow{\mathbf{C}}^{2 m}=\overrightarrow{\mathbf{b}}^{2 m}
$$

for finding coefficients $C_{\zeta, 0}^{2 m, 1}, C_{\zeta, 0}^{2 m, 2}(\zeta=1,2, \ldots, m-1)$, where a system has form as under

$$
\mathbf{D}^{2 m}=\left(\begin{array}{cc}
\mathbf{D}_{11}^{2 m} & \mathbf{0} \\
\mathbf{0} & \mathbf{D}_{22}^{2 m}
\end{array}\right),
$$

and where

$$
\begin{gathered}
\mathbf{D}_{11}^{2 m}=\left(d_{1, r \zeta}\right)_{r, \zeta=1}^{m-1}, \quad d_{1, r \zeta}=\left(-\alpha_{\zeta}^{2 m}\right)^{r-1}, \\
\mathbf{D}_{22}^{2 m}=\left(d_{2, r \zeta}\right)_{r, \zeta=1}^{m-1}, \quad d_{2, r \zeta}=-\left(\alpha_{\zeta}^{2 m}\right)^{r-1}, \\
\overrightarrow{\mathbf{C}}^{2 m \top}=\left(C_{1,0}^{2 m, 1}, \ldots, C_{m-1,0}^{2 m, 1}, C_{1,0}^{2 m, 2}, \ldots, C_{m-1,0}^{2 m, 2}\right) \\
\overrightarrow{\mathbf{b}}^{2 m \top}=\left(p_{1}^{2 m}, \ldots, p_{m-1}^{2 m}, q_{1}^{2 m}, \ldots, q_{m-1}^{2 m}\right), \\
p_{i}^{2 m}=-D^{i} \bar{\psi}_{2 m, 0}(0), \quad q_{i}^{2 m}=D^{i} \bar{\psi}_{2 m, 0}(\bar{r}), \quad i=0,1, \ldots, 2 m-1,
\end{gathered}
$$

are block matrices.

Since the values of $\alpha_{\zeta}^{2 m}(\zeta=\overline{1,2 m-2})$ are pairwise different and the matrices $\mathbf{D}_{11}^{2 m}, \mathbf{D}_{22}^{2 m}, \mathbf{D}^{2 m}$ are non-degenerate and there is an inverse of $\mathbf{D}^{2 m}$ matrix $\left(\mathbf{D}^{2 m}\right)^{-1}$, so then the only solution of the algebraic system (14) exists and it has the form: $\overrightarrow{\mathbf{C}}^{2 m}=\left(\mathbf{D}^{2 m}\right)^{-1} \overrightarrow{\mathbf{b}}^{2 m}$.

Thus, a zero approximation of $\bar{\psi}_{2 m, 0}, \Pi_{2 m, 0} \psi, Q_{2 m, 0} \psi, \lambda_{2 m, 0}$ of the problems $A_{\varepsilon}^{2 m}$ and $B_{\varepsilon}^{2 m}$ could be constructed completely.

\subsubsection{Further construction of the asymptotic series}

We can get the systems of the equations for the problems $A_{\varepsilon}^{2 m}$ and $B_{\varepsilon}^{2 m}$ and use the additional conditions for finding the solutions $\bar{\psi}_{2 m, k}, \Pi_{2 m, k} \psi, Q_{2 m, k} \psi$ and $\lambda_{2 m, k}$ in the case $k>0$ in this form

$$
\begin{gathered}
{\left[L_{2}-\lambda_{2 m, 0}\right] \bar{\psi}_{2 m, k}=\lambda_{2 m, k} \bar{\psi}_{2 m, 0}-h_{k}^{2 m}(r),} \\
L_{2 m}^{1} \Pi_{2 m, k} \psi=g_{1 k}^{2 m}\left(\rho_{1}\right), \quad L_{2 m}^{2} Q_{2 m, k} \psi=g_{2 k}^{2 m}\left(\rho_{2}\right), \\
D^{i}\left(\bar{\psi}_{2 m, k}(0)+\Pi_{2 m, k} \psi(0)\right)=D^{i}\left(\bar{\psi}_{2 m, k}(\bar{r})+Q_{2 m, k} \psi(\bar{r})\right),
\end{gathered}
$$




$$
\begin{gathered}
\Pi_{2 m, k} \psi\left(\rho_{1}\right) \rightarrow 0, \quad Q_{2 m, k} \psi\left(\rho_{2}\right) \rightarrow 0, \quad \varepsilon \rightarrow 0, \quad k=1,2, \ldots, \quad i=0,1, \ldots, 2 m-1, \\
h_{k}^{2 m}(r)=\sum_{p=1}^{[k / 2] \leqslant 2 m} \frac{2(-1)^{p+1}}{(2 p+2) ! !} D^{2 p+2} \bar{\psi}_{2 m, k-2 p}-\sum_{p=1}^{k-1} \lambda_{2 m, p} \bar{\psi}_{2 m, k-p}, \\
g_{1 k}^{2 m}\left(\rho_{1}\right)=-\frac{v_{-1}^{1}}{\rho_{1}} \Pi_{2 m, k-1} \psi+\sum_{p=0}^{k-2}\left(\lambda_{2 m, p}-v_{p}^{1} \rho_{1}^{p}\right) \Pi_{2 m, k-p-2} \psi, \\
g_{2 k}^{2 m}\left(\rho_{2}\right)=\frac{v_{-1}^{2}}{\rho_{2}} Q_{2 m, k-1} \psi+\sum_{p=0}^{k-2}\left(\lambda_{2 m, p}-(-1)^{p} v_{p}^{2} \rho_{2}^{p}\right) Q_{2 m, k-p-2} \psi .
\end{gathered}
$$

If the parameter $\lambda$ is a simple proper value of the self-adjoint operator $A$ that acting in the Hilbert space $H\left(\Omega_{\Gamma}\right)$ and if the function $\psi \in H\left(\Omega_{\Gamma}\right)$ is the corresponding normalized eigenfunction $\|\psi\|_{H\left(\Omega_{\Gamma}\right)}=1$ then in the space $H_{1}\left(\Omega_{\Gamma}\right)\left(H_{1}\left(\Omega_{\Gamma}\right)\right.$ is an orthogonal complement to the function $\psi$ in the space $\left.H\left(\Omega_{\Gamma}\right)\right)$ and then there is the operator $A-\lambda I$ that has a bounded inverse operator $(A-\lambda I)_{H_{1}\left(\Omega_{\Gamma}\right)}^{-1}$ (pseudo-resolvent).

Hence, the equation $A \varphi-\lambda \varphi=\omega \psi-h, h \in H\left(\Omega_{\Gamma}\right)$ can be solved and the solution of this equation could be presented as under

$$
\omega=(h, \psi)_{H\left(\Omega_{\Gamma}\right)}, \varphi=(A-\lambda I)_{H_{1}\left(\Omega_{\Gamma}\right)}^{-1}(\omega \psi-h),
$$

where $(\omega \psi-h) \in H_{1}\left(\Omega_{\Gamma}\right)$.

Thus, we can get the solutions $\bar{\psi}_{2 m, k, n}$ and $\lambda_{2 m, k, n}$ for any $k>0$

$$
\begin{gathered}
\lambda_{2 m, k, n}=\left(h_{k}^{2 m}, \psi_{0, n}\right)_{H\left(\Omega_{\Gamma}\right)}=\int_{\Omega_{\Gamma}} h_{k}^{2 m}(r) \psi_{0, n}(r) d r, \quad n=1,2, \ldots, \\
\bar{\psi}_{2 m, k, n}=\left(L_{2}-\lambda_{2 m, 0, n}\right)_{H_{1}\left(\Omega_{\Gamma}\right)}^{-1} h_{k}^{2 m},
\end{gathered}
$$

where $H_{1}\left(\Omega_{\Gamma}\right)$ is the orthogonal complement to eigenfunctions $\psi_{0, n} \in H\left(\Omega_{\Gamma}\right)$, ( $\Gamma=A, B)$ of the degenerate boundary value problem $A_{0}$ or $B_{0}$, where $\left\|\psi_{0, n}\right\|_{H\left(\Omega_{\Gamma}\right)}=1$.

We can find the boundary functions $\Pi_{2 m, k} \psi\left(\rho_{1}\right), Q_{2 m, k} \psi\left(\rho_{2}\right)$ for $k>0$ from the boundary value problems in the form

$$
\begin{gathered}
L_{2 m}^{1} \Pi_{2 m, k} \psi=g_{1 k}^{2 m}, \quad L_{2 m}^{2} Q_{2 m, k} \psi=g_{2 k}^{2 m}, \\
D^{i} \Pi_{2 m, k} \psi(0)-D^{i} Q_{2 m, k} \psi(\bar{r})=-D^{i} \bar{\psi}_{2 m, k}(0)+D^{i} \bar{\psi}_{2 m, k}(\bar{r}), \\
\Pi_{2 m, k} \psi\left(\rho_{1}\right) \rightarrow 0, \quad Q_{2 m, k} \psi\left(\rho_{2}\right) \rightarrow 0, \varepsilon \rightarrow 0, \quad i=0,1, \ldots, 2 m-1 .
\end{gathered}
$$

We can write the functions $\Pi_{2 m, k} \psi$ and $Q_{2 m, k} \psi$ as under

$$
\begin{aligned}
& \Pi_{2 m, k} \psi\left(\rho_{1}\right)=\Pi_{2 m, k} \tilde{\psi}\left(\rho_{1}\right)+\Pi_{2 m, k} \psi^{*}\left(\rho_{1}\right), \\
& Q_{2 m, k} \psi\left(\rho_{2}\right)=Q_{2 m, k} \tilde{\psi}\left(\rho_{2}\right)+Q_{2 m, k} \psi^{*}\left(\rho_{2}\right),
\end{aligned}
$$


where

$$
\begin{aligned}
\Pi_{2 m, k} \tilde{\psi}\left(\rho_{1}\right) & =\sum_{\zeta=1}^{m-1} C_{\zeta, k}^{2 m, 1} \exp \left(-\alpha_{\zeta}^{2 m} \rho_{1}\right), \\
Q_{2 m, k} \tilde{\psi}\left(\rho_{2}\right) & =\sum_{\zeta=1}^{m-1} C_{\zeta, k}^{2 m, 2} \exp \left(-\alpha_{\zeta}^{2 m} \rho_{2}\right)
\end{aligned}
$$

are the general solutions of the homogeneous equations (15), (17), and

$$
\begin{aligned}
& \Pi_{2 m, k} \psi^{*}\left(\rho_{1}\right)=\sum_{\zeta=1}^{m-1} \bar{C}_{\zeta, k}^{2 m, 1}\left(\rho_{1}\right) \exp \left(-\alpha_{\zeta}^{2 m} \rho_{1}\right), \\
& Q_{2 m, k} \psi^{*}\left(\rho_{2}\right)=\sum_{\zeta=1}^{m-1} \bar{C}_{\zeta, k}^{2 m, 2}\left(\rho_{2}\right) \exp \left(-\alpha_{\zeta}^{2 m} \rho_{2}\right),
\end{aligned}
$$

are the partial solutions of these inhomogeneous equations.

Since, the roots $\alpha_{i}^{2 m}$ are pairwise distinct, then the Vronsky determinants

$$
W\left[e^{-\alpha_{1}^{2 m} \rho_{1}}, \ldots, e^{-\alpha_{m-1}^{2 m} \rho_{1}}\right], W\left[e^{-\alpha_{1}^{2 m} \rho_{2}}, \ldots, e^{-\alpha_{m-1}^{2 m} \rho_{2}}\right],
$$

that are composed of the function systems $\left[\exp \left(-\alpha_{\zeta}^{2 m} \rho_{1}\right)\right]_{\zeta=1}^{m-1}$ and $\left[\exp \left(-\alpha_{\zeta}^{2 m} \rho_{2}\right)\right]_{\zeta=1}^{m-1}$, are non-zero.

Using the method of constant variation, we can find the partial solutions of the inhomogeneous equations (15), (17), i.e.

$$
\begin{gathered}
\mathbf{D}_{11}^{2 m} \vec{\Omega}_{1}=\mathbf{F}_{1}, \quad \mathbf{D}_{22}^{2 m} \vec{\Omega}_{2}=\mathbf{F}_{2}, \\
\vec{\Omega}_{1}^{\top}=\left(\frac{d \bar{C}_{\zeta, 1}^{2 m, 1}\left(\rho_{1}\right)}{d \rho_{1}}, \ldots, \frac{d \bar{C}_{\zeta, m-1}^{2 m, 1}\left(\rho_{1}\right)}{d \rho_{1}}\right), \\
\vec{\Omega}_{2}^{\top}=\left(\frac{d \bar{C}_{\zeta, 1}^{2 m, 2}\left(\rho_{2}\right)}{d \rho_{2}}, \ldots, \frac{d \bar{C}_{\zeta, m-1}^{2 m, 2}\left(\rho_{2}\right)}{d \rho_{2}}\right), \\
\mathbf{F}_{1}^{\top}=\left(0, \ldots, 0, g_{1 k}^{2 m}\right), \quad \mathbf{F}_{2}^{\top}=\left(0, \ldots, 0, g_{2 k}^{2 m}\right),
\end{gathered}
$$

where det $\left|\mathbf{D}_{11}^{2 m}\right| \neq 0$, det $\left|\mathbf{D}_{22}^{2 m}\right| \neq 0$.

We can find the functions $\bar{C}_{\zeta, k}^{2 m, 1}\left(\rho_{1}\right)$ and $\bar{C}_{\zeta, k}^{2 m, 2}\left(\rho_{2}\right)$ from the systems as under $\vec{\Omega}_{1}=\left(\mathbf{D}_{11}^{2 m}\right)^{-1} \mathbf{F}_{1}, \vec{\Omega}_{2}=\left(\mathbf{D}_{22}^{2 m}\right)^{-1} \mathbf{F}_{2}$.

After integrating and substituting the solutions in (18), (19), we can find as many arbitrary constants as the boundary conditions of the problems $A_{\varepsilon}^{2 m}$ or $B_{\varepsilon}^{2 m}$ fall out when we proceed to analysis of the degenerate problems $A_{0}$ or $B_{0}$.

Thus, this algorithm allows us to find the asymptotic solutions of the problems $A_{\varepsilon}^{2 m}$ and $B_{\varepsilon}^{2 m}$ with any desired degree of accuracy of a small parameter $\varepsilon^{j}$. 


\section{Asymptotic analysis of the solutions}

We can formulate the following theorem for the justification of the asymptotic solutions of the problems $A_{\varepsilon}^{2 m}$ and $B_{\varepsilon}^{2 m}$.

Theorem 1. If the self-adjoint elliptic operators $L_{2}, L_{2 m}$ satisfy Conditions 1-3 for the problems $A_{\varepsilon}^{2 m}, B_{\varepsilon}^{2 m}, A_{0}, B_{0}$ and the function $v(r) \in C^{\infty}$ is represented as the uniformly converging series in the neighborhood of the point $r=0$ and the neighborhood of the point $r=r_{0}$

$$
v(r)=\sum_{s=-1}^{\infty} v_{s}^{1} r^{s}, \quad v(r)=\sum_{s=-1}^{\infty} v_{s}^{2}\left(r-r_{0}\right)^{s},
$$

the asymptotic solutions of boundary value problems $A_{\varepsilon}^{2 m}$ and $B_{\varepsilon}^{2 m}$ exist.

The corresponding $n$ eigenvalue $\lambda_{\varepsilon, 2 m, n}$ and the corresponding $n$ eigenfunction $\psi_{\varepsilon, 2 m, n}(r)$ of the operator $\widetilde{L}_{2 m}$ have the following asymptotic representations

$$
\lambda_{\varepsilon, 2 m, n} \equiv \lambda_{2 m, 0, n}+\varepsilon \lambda_{2 m, 1, n}+\varepsilon^{2} \lambda_{2 m, 2, n}+\ldots+\varepsilon^{j+1} \Delta_{j}^{2 m},
$$

$\psi_{\varepsilon, 2 m, n}(r)=\sum_{k=0}^{\infty} \varepsilon^{j}\left(\bar{\psi}_{2 m, k, n}(r)+\Pi_{2 m, k, n} \psi\left(\rho_{1}\right)+Q_{2 m, k, n} \psi\left(\rho_{2}\right)\right)+\varepsilon^{j+1} z_{j}^{2 m}(r)$,

where $\lambda_{2 m, 0, n}=\lambda_{0, n}$ is $n$-th simple eigenvalue and $\bar{\psi}_{2 m, 0, n}(r)=\psi_{0, n}$ is the $n$-th function of the operator $L_{2}$ for boundary value problems $A_{0}$ and $B_{0}$; the functions $\bar{\psi}_{2 m, k, n}(r), \Pi_{2 m, k, n} \psi, Q_{2 m, k, n} \psi$ and the values of $\lambda_{2 m, k, n}$ for $k>0$ are determined from the systems of the equations and the boundary conditions given in Paragraph 2.

The estimations for the residual members $\bar{z}_{j}^{2 m}(r)$ and $\Delta_{j}^{2 m}$ have form as under $\left\|D \bar{z}_{j}^{2 m}\right\|_{H}+\left\|\bar{z}_{j}^{2 m}\right\|_{H}=O\left(\varepsilon^{j+1}\right), \Delta_{j}^{2 m}=O(1)$, for p-order derivative of the partial sum $\Theta_{j} \psi_{\varepsilon, 2 m, n}$ is $\left\|D^{q+2} \bar{z}_{j}^{2 m}\right\|_{H}=O\left(\varepsilon^{j-q+1}\right), 1 \leqslant q \leqslant s, s \geqslant 2 m-2$, in the inner subdomain $\left[\delta, r_{0}-\delta\right]$ is $\left\|D^{q+2} \bar{z}_{j}^{2 m}\right\|_{H}=O\left(\varepsilon^{j+1}\right),|q| \leqslant s$, in border regions $(0, \delta]$ and $\left[r_{0}-\delta, r_{0}\right)$ is $\left\|D^{q+2} \bar{z}_{j}^{2 m}\right\|_{H}=O\left(\varepsilon^{j-q+1}\right), 1 \leqslant|q| \leqslant s$.

Proof. It is assumed that the function $\Theta_{j} \psi_{\varepsilon, 2 m, n}(r)$ satisfies the boundary conditions of the problems $A_{\varepsilon}^{2 m}$ and $B_{\varepsilon}^{2 m}$ and

$$
\left\|\psi_{0}\right\|_{H}=\left\|\bar{\psi}_{2 m, 0}\right\|_{H}=1, \quad\left\|\psi_{\varepsilon, 2 m}\right\|_{H}=1+O(\varepsilon) .
$$

Using series for the constructions of a solution, we can get

$$
\left[\widetilde{L}_{2 m}^{\varepsilon}-\Theta_{j} \lambda_{\varepsilon, 2 m}\right] \Theta_{j} \psi_{\varepsilon, 2 m}(r)=\varepsilon^{j+1} \bar{f}_{j}^{2 m},
$$

where $\bar{f}_{j}^{2 m}$ is the restricted function $\left(\left\|\bar{f}_{j}^{2 m}\right\|_{H}=O(1)\right)$.

According to the estimate, we have the evaluation in the form as under

$$
\inf _{n}\left|\lambda-\lambda_{\varepsilon, 2 m, n}\right| \leqslant\left\|\widetilde{L}_{2 m}^{\varepsilon} \psi-\lambda \psi\right\|_{H} /\|\psi\|_{H},
$$


where $\psi \in \Omega_{\Gamma}$ is an arbitrary function from the scope of the operator $\widetilde{L}_{2 m}^{\varepsilon}$ and $\lambda>0$ is an arbitrary real number.

Using the evaluation $\left\|\psi_{\varepsilon, 2 m}\right\|_{H}=1+O(\varepsilon)$, we can get that

$$
\lambda_{\varepsilon, 2 m, n}-\Theta_{j} \lambda_{\varepsilon, 2 m}=\varepsilon^{j+1} \Delta_{j}^{2 m},
$$

where $\left|\Delta_{j}^{2 m}\right| \leqslant\left\|\bar{f}_{j}\right\|_{H} /\left\|\Theta_{j} \psi_{\varepsilon, 2 m}\right\|_{h}$. Hence, we can get the estimate $\Delta_{j}^{2 m}=$ $O(1)$.

Let $T_{d}^{0}$ be a closed linear shell consisting of eigenfunctions $\Theta_{j} \psi_{\varepsilon, 2 m, n}(r)$, corresponding to the corresponding eigenvalues $\Theta_{j} \lambda_{\varepsilon, 2 m, n}$, that are lying on a segment $\left[\lambda_{0, n}-d, \lambda_{0, n}+d\right]$, where $d$ is a number $d>\Sigma\left(\| \tilde{l}_{2 m}^{\varepsilon} \theta_{j} \psi_{\varepsilon, 2 m}-\right.$ $\left.\Theta_{j} \lambda_{\varepsilon, 2 m} \Theta_{j} \psi_{\varepsilon, 2 m} \|_{H} \leqslant \sigma\right)$, then there is such a function $\tilde{\psi}_{T} \in T_{d}^{0},\left\|\tilde{\psi}_{T}\right\|_{H}=1$, for which the following inequality $\left\|\Theta_{j} \psi_{\varepsilon, 2 m}-\tilde{\psi}_{t}\right\|_{h} \leqslant 2 \Sigma / d$ is satisfied.

If $\varepsilon$ is sufficiently small, then the following inequalities occur

$$
\lambda_{\varepsilon, 2 m, n-1}-\lambda_{0, n-1} \leqslant d, \quad \lambda_{\varepsilon, 2 m, n}-\lambda_{0, n} \leqslant d, \quad \lambda_{\varepsilon, 2 m, n+1}-\lambda_{0, n+1} \leqslant d,
$$

where $3 d=\min \left[\lambda_{0, n}-\lambda_{0, n-1} ; \lambda_{0, n+1}-\lambda_{0, n}\right]$.

Thus, a segment $\left[\lambda_{0, n}-d, \lambda_{0, n}+d\right]$ contains the single eigenvalue $\lambda_{\varepsilon, 2 m, n}$ of the operator $\widetilde{L}_{2 m}^{\varepsilon}$, which is relevant to the single normalized eigenfunction $\psi_{\varepsilon, 2 m, n}(r)$, which coinciding with the normalized function $\tilde{\psi}_{T}$, and there is the estimation

$$
\left\|\psi_{\varepsilon, 2 m, n}-\Theta_{j} \psi_{\varepsilon, 2 m, n} /\right\| \Theta_{j} \psi_{\varepsilon, 2 m, n}\left\|_{H}\right\|_{H} \leqslant O\left(\varepsilon^{j+1}\right) .
$$

Thus, we can get the estimation $\left\|z_{j}^{2 m}\right\|_{H}=o(1)$, where $\bar{z}_{j}^{2 m}=\varepsilon^{j+1} z_{j}^{2 m}=$ $\bar{\psi}_{\varepsilon, 2 m, n}-\theta_{j} \psi_{\varepsilon, 2 m, n}$, and $\bar{\psi}_{\varepsilon, 2 m, n}=\left\|\Theta_{j} \psi_{\varepsilon, 2 m, n}\right\|_{H} \psi_{\varepsilon, 2 m, n}$.

Since the inequality $\lambda_{\varepsilon, 2 m, \gamma} \geqslant \lambda_{0, \gamma}, \gamma=1,2, \ldots$ is true and there is the ratio

$$
\left[\widetilde{L}_{2 m}^{\varepsilon}-\Theta_{j} \lambda_{\varepsilon, 2 m}\right] \Theta_{j} \psi_{\varepsilon, 2 m}(r)=\varepsilon^{j+1} \bar{f}_{j}^{2 m}, \quad\left\|\bar{f}_{j}^{2 m}\right\|_{H}=o(1)
$$

we can get the following estimations

$$
\left\|\left[\widetilde{L}_{2 m}^{\varepsilon}-\lambda_{\varepsilon, 2 m}\right] \bar{z}_{j}^{2 m}\right\|_{H}=O\left(\varepsilon^{j+1}\right)
$$

and $\left\|\widetilde{L}_{2 m}^{\varepsilon} \bar{z}_{j}^{2 m}\right\|_{H} \leqslant\left\|\left[\widetilde{L}_{2 m}^{\varepsilon}-\lambda_{\varepsilon, 2 m}\right] \bar{z}_{j}^{2 m}\right\|_{H}+\left|\lambda_{\varepsilon, 2 m}\right|\left\|\bar{z}_{j}^{2 m}\right\|_{h}=o\left(\varepsilon^{j+1}\right)$.

Using Conditions $1-3$ and assuming that the function $\bar{z}_{j}^{2 m}$ satisfies the boundary conditions of the problems $A_{\varepsilon}^{2 m}$ and $B_{\varepsilon}^{2 m}$, we can get the following estimations

$$
\left\|\bar{z}_{j}^{2 m}\right\|_{H}^{2} \leqslant \sum_{p=1}^{m-1} \varepsilon^{2 p}\left\|D^{p} \bar{z}_{j}^{2 m}\right\|_{H}^{2}+\left\|D \bar{z}_{j}^{2 m}\right\|_{H}^{2}+\left\|\bar{z}_{j}^{2 m}\right\|_{h}^{2} \leqslant \bar{C} \varepsilon^{2(j+1)}\left\|\bar{W}_{j}^{2 m}\right\|_{h}^{2},
$$


where the constant $\bar{C}>0$ which is independent of $r$ and $\varepsilon$ and the function $\bar{w}_{j}^{2 m}$ is the restricted function for which the estimation $\left\|\bar{w}_{j}^{2 m}\right\|_{H}=O(1)$ takes place.

This implies the estimate for $\bar{z}_{j}^{2 m}$ that is in the conditions of the theorem. $\square$

\section{Solutions behavior analysis of the problems $A_{\varepsilon}^{2 m}$ and $B_{\varepsilon}^{2 m}$ in the case $m \rightarrow \infty$}

Here we investigate the question about the behavior of the eigenfunctions and the eigenvalues of $A_{\varepsilon}^{2 m}$ and $B_{\varepsilon}^{2 m}$ problems in the case of unlimited increasing of $2 m$-order LTKT-equation.

Let's consider the problems of $A_{\varepsilon}^{2 m}, B_{\varepsilon}^{2 m}$ and $A_{\varepsilon}^{2 m+2}, B_{\varepsilon}^{2 m+2}$ for finding $\left[\psi_{\varepsilon, 2 m, \gamma}\right]_{\gamma=1}^{\infty},\left[\lambda_{\varepsilon, 2 m, \gamma}\right]_{\gamma=1}^{\infty}$ and $\left[\psi_{\varepsilon, 2 m+2, \gamma}\right]_{\gamma=1}^{\infty},\left[\lambda_{\varepsilon, 2 m+2, \gamma}\right]_{\gamma=1}^{\infty}$. Here we assume that the eigenvalues are arranged in order of monotonic increase.

Let the relations

$$
\Delta_{2 m}^{2 m+2} \psi_{\varepsilon, n}=\psi_{\varepsilon, 2 m+2, n}-\psi_{\varepsilon, 2 m, n}, \quad \Delta_{2 m}^{2 m+2} \lambda_{\varepsilon, n}=\lambda_{\varepsilon, 2 m+2, n}-\lambda_{\varepsilon, 2 m, n},
$$

take place, where $\left\|\psi_{\varepsilon, 2 m+2}\right\|_{H}=1,\left\|\psi_{\varepsilon, 2 m}\right\|_{H}=1$.

We can formulate the following

Theorem 2. If the positive self-adjoint elliptic operators act in the space $H\left(\Omega_{\Gamma}\right), L_{2}, L_{2 m}$ and satisfy Conditions 1-3 for the problems $A_{\varepsilon}^{2 m}, B_{\varepsilon}^{2 m}, A_{0}$, $B_{0}$, then we have the following estimates for $m \rightarrow \infty$

$$
\begin{gathered}
\left|\Delta_{2 m}^{2 m+2} \lambda_{\varepsilon, n}\right| \leqslant\left\|\widetilde{L}_{2 m+2}^{\varepsilon}-\widetilde{L}_{2 m}^{\varepsilon}\right\|_{H} \leqslant \frac{2 \varepsilon^{2 m}}{(2 m+2) ! !}, \\
\left\|\Delta_{2 m}^{2 m+2} \psi_{\varepsilon, n}\right\|_{H} \leqslant \frac{2 \varepsilon^{2 m}}{(2 m+2) ! !} .
\end{gathered}
$$

Proof. We can get the ratios

$$
\begin{gathered}
\Delta_{2 m}^{2 m+2} L=\widetilde{L}_{2 m+2}^{\varepsilon}-\widetilde{L}_{2 m}^{\varepsilon}=\frac{2(-1)^{m+1} \varepsilon^{2 m}}{(2 m+2) ! !} D^{2 m+2}, \\
\lambda_{\varepsilon, 2 m+2, n} \leqslant \sup _{\varphi}\left[\left(\left(\Delta_{2 m}^{2 m+2} L+\widetilde{L}_{2 m}^{\varepsilon}\right) \varphi, \varphi\right)_{H}\right] \leqslant \lambda_{\varepsilon, 2 m, n}+\bar{\lambda}, \\
\|\varphi\|_{H}=1,\left(\varphi, \psi_{\varepsilon, 2 m, \gamma}\right)_{H}=0, \quad \gamma=\overline{1, n-1},
\end{gathered}
$$

where $\bar{\lambda}$ is the largest positive eigenvalue of the operator $\Delta_{2 m}^{2 m+2} L$, and there is the following inequality $\bar{\lambda} \leqslant\left\|\Delta_{2 m}^{2 m+2} L\right\|_{H}$.

We get the inequalities $\left|\Delta_{2 m}^{2 m+2} \lambda_{\varepsilon, n}\right| \leqslant\left\|\widetilde{L}_{2 m+2}^{\varepsilon}-\widetilde{L}_{2 m}^{\varepsilon}\right\|_{H}$, where $\left|\Delta_{2 m}^{2 m+2} \lambda_{\varepsilon, n}\right| \leqslant 2 \varepsilon^{2 m} /(2 m+2) ! !$. 
Thus, there is the equality

$$
\left[\widetilde{L}_{2 m+2}^{\varepsilon}-\lambda_{\varepsilon, 2 m+2}\right] \Delta_{2 m}^{2 m+2} \psi_{\varepsilon}=\frac{2 \varepsilon^{2 m}}{(2 m+2) ! !} \bar{v}_{2 m},
$$

where $\bar{v}_{2 m}$ is the restricted function, $\left\|\bar{v}_{2 m}\right\|_{H}=O(1),\left(\bar{v}_{2 m}, \Delta_{2 m}^{2 m+2} \psi_{\varepsilon}\right)_{H}=0$.

We can assume that the operator $\left(\widetilde{L}_{2 m+2}^{\varepsilon}-\lambda_{\varepsilon, 2 m+2}\right)$ has a limited inverse operator $\left(\widetilde{L}_{2 m+2}^{\varepsilon}-\lambda_{\varepsilon, 2 m+2}\right)_{h_{1}}^{-1}$ (a pseudo-resolvent) and there are the ratios

$$
\Delta_{2 m}^{2 m+2} \psi_{\varepsilon}=\frac{2 \varepsilon^{2 m}}{(2 m+2) ! !}\left(\widetilde{L}_{2 m+2}^{\varepsilon}-\lambda_{\varepsilon, 2 m+2}\right)_{H_{1}}^{-1} \bar{v}_{2 m}
$$

and $\left\|\Delta_{2 m}^{2 m+2} \psi_{\varepsilon, n}\right\|_{H} \leqslant \frac{2 \varepsilon^{2 m}}{(2 m+2) ! !}$. Thus, the theorem is proved.

\section{Construction of an asymptotic solution in the case of the oscillator potential}

We can consider the boundary value problem $B_{\varepsilon}^{2 m}$ on the $[0, \infty+)$ axis with the quasi-potential of a linear harmonic oscillator in the form $v(r)=r^{2}$. Analysis of this problem allows to describe the behavior chains of harmonic oscillators with periodic boundary conditions when they are very far apart from each other.

The solution of the degenerate boundary value problem $B_{0}$ is an orthonormal system of Hermite functions

$$
\psi_{0, n}=\left[n ! 2^{n} \sqrt{\pi}^{-1 / 2} \exp \left(-r^{2} / 2\right) H_{n}(r), \quad \lambda_{n}=2 n+1, \quad n=1,3,5, \ldots,\right.
$$

where

$$
H_{n}(r)=n ! \sum_{m=0}^{[n / 2]}(-1)^{m} \frac{2 r^{n-2 m}}{m !(n-2 m) !} .
$$

We can show that the zero approximation has equality $\bar{\psi}_{2 m, 0, n}=\psi_{0, n}$.

We can find the functions $\Pi_{2 m, 0, n} \psi\left(\rho_{1}\right)$ and $Q_{2 m, 0, n} \psi\left(\rho_{2}\right)$ in the form

$$
\begin{gathered}
\Pi_{2 m, 0, n} \bar{\psi}\left(\rho_{1}\right)=\sum_{k=1}^{m-1} C_{0 k n} \exp \left(-\alpha_{k} \rho_{1}\right), \quad Q_{2 m, 0, n} \psi\left(\rho_{2}\right)=0, \\
C_{0 k n}=\sum_{s=1}^{m-1} \varepsilon^{s-1} A_{k, s} \frac{d^{s} \bar{\psi}_{2 m, 0, n}(0)}{d r^{s}}, \\
A_{1, s}=\frac{1}{\prod_{l \neq s}\left(\alpha_{l}-\alpha_{s}\right)}, \quad l, s=1, \ldots, m-1,
\end{gathered}
$$




$$
\begin{gathered}
A_{2 q, s}=A_{2 q+1, s}=-\frac{\sum_{r=1}^{2 q} \frac{(-1)^{r}}{(2 r) !} \alpha_{s}^{2 r-2}}{\prod_{l \neq s}\left(\alpha_{l}-\alpha_{s}\right)}, q=1,2, \ldots,(m-1) / 2, l, s=1, \ldots, m-1, \\
D^{i} \bar{\psi}_{0, n}(0)=\left.\left[n ! 2^{n} \sqrt{\pi}\right]^{-1 / 2} D^{i}\left[\exp \left(-r^{2} / 2\right) H_{n}(r)\right]\right|_{(r=0)}, \quad n=1,3,5, \ldots
\end{gathered}
$$

The first approximation of the solution has the forms

$$
\begin{gathered}
\bar{\psi}_{2 m, 1, n}=\psi_{0, n}, \quad \lambda_{2 m, 1, n}=0 \\
\Pi_{2 m, 1, n} \bar{\psi}\left(\rho_{1}\right)=\sum_{k=1}^{m-1} C_{1 k n} \exp \left(-\alpha_{k} \rho\right), \quad Q_{2 m, 1, n} \psi\left(\rho_{2}\right)=0 \\
C_{1 k n}=\sum_{s=1}^{m-1} \varepsilon^{s-1} A_{k, s} \frac{d^{s} \bar{\psi}_{2 m, 1, n}(0)}{d r^{s}}
\end{gathered}
$$

The next approximation has the following ratios

$$
\begin{gathered}
\bar{\psi}_{2 m, 2, n}=\psi_{0, n}, \quad \lambda_{2 m, 2, n}=\varepsilon \frac{1}{4}\left(n^{2}+(n+1)^{2}\right), \quad n=1,3,5, \ldots, \\
\Pi_{2 m, 1, n} \bar{\psi}\left(\rho_{1}\right)=\sum_{k=1}^{m-1} F_{k n}(r, \varepsilon) \exp \left(-\varepsilon^{-1} \alpha_{k} r\right), \quad Q_{2 m, 2, n} \psi\left(\rho_{2}\right)=0 \\
F_{k n}(r, \varepsilon)=R_{k n}+\rho_{1} T_{k n}, \quad R_{k n}=C_{1 k n}-\lambda_{2 m, 0, n} \sum_{p=0}^{m-1} C_{1 p n} B_{p, k} \\
T_{k n}=-C_{1 k n} \lambda_{2 m, 0, n} \bar{B}_{k, k}, \quad \bar{B}_{k, k}=\frac{1}{\prod_{j \neq k}\left(\alpha_{j}-\alpha_{k}\right)}, \\
B_{k, k}=\frac{\sum_{j=1, j \neq k}^{m-1}\left(\alpha_{k}-\alpha_{j}\right)}{\prod_{j \neq k}\left(\alpha_{j}-\alpha_{k}\right)^{2}}, \quad B_{p, k}=\frac{1}{\left(\alpha_{p}-\alpha_{k}\right) \prod_{j \neq k}\left(\alpha_{j}-\alpha_{k}\right)} .
\end{gathered}
$$

Thus, we can continue the procedure for constructing the asymptotic series and building an asymptotic solution of the problem under consideration with accuracy up to any given order $\varepsilon$.

\section{Conclusions}

Recently, there is a great interest in studying properties of bound states of a quarkonium such as charmonium $c \bar{c}$ and bottomonium $b \bar{b}$. These states are similar to the properties of positronium (the bound state of an electron and a positron). Special attention of researchers who deal with bound states of quarks is paid to quasi-potential methods. The quasi-potential approach allows to describe the characteristics of relativistic elementary particles such as amplitudes of hadron elastic scattering, mass spectra and widths of meson decays, and the cross sections of deep inelastic scattering of leptons on 
hadrons. Since experimental measurements of relativistic elementary particles are carried out with high accuracy, the quark systems models allow to use the precision calculation of various parameters. Experiment has amassed a wealth of high precision data on quarkonium production in relativistic heavy ion collisions at RHIC and LHC in different kinematical regimes that provides a challenging testing ground for theory and phenomenology.

We use a quasi-potential approach in our work. The quasi-potential method in the field theory is based on a two-time Green function for particle systems. The bounded states of such systems are described by a wave function that satisfies a quasi-potential Schrödinger-type equation that depends on energy and non-local potential. The main advantage of this quasi-potential equation is its three-dimensional character. We have shown the absence of a non-physical parameter of relative time for this equation. This quasi-potential wave equation can be obtained for any system numbers of particles with arbitrary spins. This approach was successfully applied to calculate corrections to the energy levels of hydrogen-like systems within the framework of quantum electrodynamics. The great number of properties of the elementary particles amplitude scattering at high energies is explained using a quasi-potential Lippman-Schwinger equation with a Gaussian potential. The quasi-potential method has a number of advantages among the methods of studying the relativistic two-body problem. The advantage of this approach is that quasipotential equations are written out in three-dimensional space, which makes it possible to use the methods of non-relativistic quantum mechanics.

In this paper Sturm-Liouville problems with periodic boundary conditions on a segment and a positive half-line are formulated for the truncated to order $2 m$ relativistic finite-difference Schrödinger equation (LogunovTavkhelidze-Kadyshevsky equation, LTKT-equation) with a small parameter. For these singularly perturbed problems a method is proposed for constructing asymptotic solutions with accuracy up to any given order $\varepsilon$. With the help of this method asymptotic solutions in the form of regular and boundary-layer parts are obtained and the question of asymptotic solutions behavior when $\varepsilon \rightarrow 0$ is investigated.

The behavior of solutions is investigated in the case $m \rightarrow \infty$ and estimation of this behavior is given. It makes possible to determine the convergence of solutions of the Sturm-Liouville problems for LTKT-equation with periodic boundary conditions in the case $m \rightarrow \infty$.

In non-relativistic quantum mechanics, the particle in a one-dimensional lattice is a problem that occurs in the model of a periodic crystal lattice. The potential is caused by ions in the periodic structure of the crystal creating an electromagnetic field, so electrons are subject to a regular potential inside the lattice. This is a generalization of the free electron model, which assumes zero potential inside the lattice.

In this work the Sturm-Liouville problem on the positive half-line with a periodic boundary conditions for the quantum harmonic oscillator is considered and eigenfunctions and eigenvalues are constructed as asymptotic solutions for $2 m$-order LTKT-equation. Their solutions allow to describe the behavior chains of harmonic oscillators with periodic boundary conditions when they are very far apart from each other. We can use more complex quasi-potentials and describe the bounded states of the elementary particles in the quark-gluon plasma. 


\section{Acknowledgments}

The publication has been prepared with the support of the "RUDN University Program 5-100" and funded by RFBR according to the research projects No. 18-07-00567.

\section{References}

[1] A. A. Atanasov and A. T. Marinov, " $\hbar-$ Expansion for bound states described by the relativistic three-dimensional two-particle quasi-potential equation," Theoretical and Mathematical Physics, vol. 129, no. 1, pp. 14001407, 2001. DOI: 10.1023/A:1012423629038.

[2] Y. D. Chernichenko, "On a solution of the relativistic inverse problem for the sum of nonlocal separable quasipotentials," Russian Physics Journal, vol. 55, no. 6, pp. 699-711, 2012. DOI: 10.1007/s11182-012-9869-3.

[3] V. G. Kadyshevsky, R. M. Mir-Kasimov, and N. B. Skachkov, "Quasipotential Approach and the Expansion in Relativistic Spherical Functions," Nuovo Cimento A, vol. 55, no. 2, pp. 233-257, 1968. DOI: 10.1007 / BF02759225.

[4] V. G. Kadyshevsky and M. Mateev, "On a Relativistic Quasipotential Equation in the Case of Particles with Spin," Nuovo Cimento A, vol. 55, no. 2, pp. 275-300, 1968. DOI: 10.1007/BF02759227.

[5] V. G. Kadyshevsky, "Quasipotential type equation for the relativistic scattering amplitude," Nuclear Physics, no. 1, pp. 125-148, 1968.

[6] A. A. Logunov and A. N. Tavkhelidze, "Quasi-optical approach in quantum field theory," Nuovo Cimento, vol. 29, pp. 380-399, 1963. DOI: 10.1007/BF02750359.

[7] A. A. Logunov and A. N. Tavkhelidze, "Quasi-potential character of the scattering amplitude," Nuovo Cimento, vol. 30, pp. 134-142, 1963. DOI: $10.1007 /$ BF02750754.

[8] A. A. Logunov, A. N. Tavkhelidze, and O. A. Khrustalev, "Quasipotential character of the Mandelstam representation," Physics Letters, vol. 4, no. 6, pp. 325-326, 1963.

[9] V. A. Matveev, V. I. Savrin, A. N. Sissakian, and A. N. Tavkhelidze, "Relativistic Quark Models in the Quasipotential Approach," Theoretical and Mathematical Physics, vol. 132, no. 2, pp. 1119-1136, 2002. DoI: 10.1023/A:1019704709192.

[10] A. Pokraka and R. Dick, "Dimensional effects on the density of states in systems with quasi-relativistic dispersion relations and potential wells," Canadian Journal of Physics, vol. 94, no. 8, pp. 773-779, 2016. DOI: 10.1139/cjp-2015-0758.

[11] K. A. Sveshnikov and P. K. Silaev, "Quasi-exact solution of a relativistic finite-difference analogue of the Schrödinger equation for a rectangular potential well," Theoretical and Mathematical Physics, vol. 132, no. 3, pp. 408-433, 2002. DOI: 10.1023/A : 1020220104534. 
[12] K. A. Sveshnikov and P. K. Silaev, "Quasi-exact solution of the problem of relativistic bound states in the $(1+1)$-dimensional case," Theoretical and Mathematical Physics, vol. 149, no. 3, pp. 1665-1689, 2006. DOI: $10.1007 / \mathrm{s} 11232-006-0150-1$.

[13] V. S. Minh, E. P. Zhidkov, and V. G. Kadyshevsky, "Solutions of relativistic radial quasipotential equations," Theoretical and Mathematical Physics, vol. 63, no. 2, pp. 493-503, 1985. DOI: 10.1007/BF01017906.

[14] I. V. Amirkhanov, E. P. Zhidkov, I. E. Zhidkova, and S. A. Vasilyev, "Construction of an asymptotic approximation of eigenfunctions and eigenvalues of a boundary value problem for the singular perturbed relativistic analog of the Schrödinger equation with an arbitrary potential [Asimptotika sobstvennyh funkcij i sobstvennyh znachenij kraevoj zadachi dlya singulyarno vozmushchennogo relyativistskogo analoga uravneniya Schrödingera pri proizvol'nom potenciale]," Mathematical Models and Computer Simulations [Matematicheskoe modelirovanie], vol. 15, no. 9, pp. 3-16, 2003, in Russian.

[15] I. V. Amirkhanov, E. P. Zhidkov, D. Z. Muzafarov, N. R. Sarker, I. Sarhadov, and Z. A. Sharipov, "Investigation of boundary-value problems for the singular perturbed differential equation of high order [Issledovanie kraevyh zadach dlya singulyarno-vozmushchennogo differencial'nogo uravneniya vysokogo poryadka]," Mathematical Models and Computer Simulations [Matematicheskoe modelirovanie], vol. 19, no. 11, pp. 65-79, 2007, in Russian.

[16] I. V. Amirkhanov, N. R. Sarker, I. Sarhadov, Z. K. Tukhliev, and Z. A. Sharipov, "Analytical and Computational Investigations of Solutions of Boundary-Value Problems for the Quasipotential Equation [Analiticheskoe i chislennoe issledovaniya reshenij kraevyh zadach dlya kvazipotencial'nogo uravneniya]," Bulletin of Peoples' Friendship University of Russia. Series Mathematics. Information Sciences. Physics /Vestnik Rossijskogo universiteta druzhby narodov. Seriya: Matematika, informatika, fizika], no. 4, pp. 44-52, 2012, in Russian.

[17] V. O. Galkin, R. N. Faustov, and D. Ebert, "Logunov-Tavkhelidze equation in the relativistic quark model," Theoretical and Mathematical Physics, vol. 191, no. 2, pp. 641-648, 2017. DOI: 10 . 1134 / S0040577917050038.

[18] N. Alam and S. Mandal, "On the quantum phase fluctuations of coherent light in a chain of two anharmonic oscillators coupled through a linear one," Optics Communications, vol. 366, pp. 340-348, 2016. DOI: 10. 1016/j.optcom. 2016.01.019.

[19] Z. Chen, "Mapping quantum many-body system to decoupled harmonic oscillators: general discussions and examples," Physics Letters A, vol. 382, no. 37, pp. 2613-2617, 2018. DOI: 10.1016/j . physleta.2018.07.043.

[20] E. P. Zhidkov, V. G. Kadyshevsky, and Y. V. Katyshev, "Problem of the $c \rightarrow \infty$ limit in the relativistic Schrödinger equation," Theoretical and Mathematical Physics, vol. 3, no. 2, pp. 443-446, 1970. DOI: 10. 1007/BF01046508. 


\section{For citation:}

I. V. Amirkhanov, I. S. Kolosova, S. A. Vasilyev, Asymptotic solution of SturmLiouville problem with periodic boundary conditions for relativistic finitedifference Schrödinger equation, Discrete and Continuous Models and Applied Computational Science 28 (3) (2020) 230-251. DOI: 10.22363/2658-46702020-28-3-230-251.

\section{Information about the authors:}

Amirkhanov, Ilkizar V. - Candidate of Physical and Mathematical Sciences, head of the group of Methods for Solving Mathematical Physics Problems of Laboratory of Information Technologies (LIT) of Joint Institute for Nuclear Research (e-mail: camir@jinr.ru, phone: +7(496)2162547, ORCID: https://orcid.org/0000-0003-2621-144X, Scopus Author ID: 6507929197)

Kolosova, Irina S. - PhD's degree student of Department of Applied Probability and Informatics of Peoples' Friendship University of Russia (RUDN University) (e-mail: i.se.kolosova@gmail.com, phone: $+7(495) 9522823$, ORCID: https://orcid.org/0000-0002-7594-3375)

Vasilyev, Sergey A. - Candidate of Physical and Mathematical Sciences, assistant professor of Department of Applied Probability and Informatics of Peoples' Friendship University of Russia (RUDN University) (e-mail: vasilyev-sa@rudn.ru, phone: $+7(495) 9522823$, ORCID: https://orcid.org/0000-0003-1562-0256, ResearcherID: 5806-2016, Scopus Author ID: 56694334800) 
УДК 517.958, 517.963

PACS 02.30.Hq, 02.30.Mv, 03.65.Ge, 11.10.Jj, 03.65.Pm, 02.30.Em

DOI: $10.22363 / 2658-4670-2020-28-3-230-251$

\title{
Асимптотическое решение задачи Штурма-Лиувилля с периодическими граничными условиями для релятивистского конечно-разностного уравнения Шрёдингера
}

\author{
И. В. Амирханов ${ }^{1}$, И. С. Колосова ${ }^{2}$, С. А. Васильев ${ }^{2}$ \\ ${ }^{1}$ Объединённый институт ядерных исследований \\ ул. Жолио-Кюри, д. 6, Дубна, Московская область, Россия, 141980 \\ 2 Российский университет дружбы народов \\ ул. Миклухо-Маклая, д. 6, Москва, Россия, 117198
}

Описание взаимодействия релятивистских частиц в рамках квазипотенциального подхода широко применяется в современной физике. Этот подход основан на так называемой ковариантной формулировке квантовой теории поля, в которой эта теория рассматривается на пространственно-подобной трёхмерной гиперповерхности в пространстве Минковского. Особое внимание в этом подходе уделяется методам построения различных квазипотенциалов, а также использованию квазипотенциального подхода для описания характеристик связанных состояний в кварковых моделях, таких как амплитуды адронного упругого рассеяния, масс-спектры и ширины распадов мезонов, сечения глубокого неупругого рассеяния лептонов на адронах.

В настоящей работе сформулированы задачи Штурма-Лиувилля с периодическими граничными условиями на отрезке и на положительной полупрямой для усечённого релятивистского конечно-разностного уравнения Шрёдингера (уравнение Логунова-Тавхелидзе-Кадышевского, LTKT-уравнение) с малым параметром при старшей производной.

Целью работы является построение асимптотических решений (собственных функций и собственных значений) в виде регулярных и погранслойных частей решений для этой сингулярно возмущённой задачи Штурма-Лиувилля. Основная задача исследования состоит в асимптотическом анализе поведенческих решений рассматриваемой задачи в случае $\varepsilon \rightarrow 0$ и $m \rightarrow \infty$. Нами был предложен метод построения асимптотических решений (собственных функций и собственных значений), который является обобщением асимптотических методов решения сингулярно возмущённых задач, представленных в работах А.Н. Тихонова, А. Б. Васильевой и В. Ф. Бутузова. Основной результат данной работы - доказанные теоремы об асимптотической сходимости решений сингулярно возмущённой задачи к решениям вырожденной задач при $\varepsilon \rightarrow 0$ и сходимости решений усечённого LTKT-уравнения в случае $m \rightarrow \infty$. Kроме того, в статье нами рассматривается задача Штурма-Лиувилля на положительной полуоси для LTKT-уравнения 4-го порядка с периодическими граничными условиями для квантового гармонического осциллятора. Для этой задачи построены асимптотические приближения собственных функций и собственных значений и показана их сходимость к решению вырожденной задачи.

Ключевые слова: асимптотический анализ, сингулярно возмущённое дифференциальное уравнение, задача Штурма-Лиувилля, релятивистское конечно-разностное уравнение Шрёдингера, периодические краевые условия, квазипотенциальный подход 